\title{
Heating of Protons by Resonant Absorption in a Multicomponent Plasma 2. Theoretical Model
}

\author{
Dominique Le Quéau, ${ }^{1}$ Alain Roux, ${ }^{2}$ Jean Louis Rauch, ${ }^{3}$ François Lefeuvre, ${ }^{3}$ \\ AND JEAN Michel BosQUed ${ }^{4}$
}

\begin{abstract}
A theory is proposed to explain the selective heating of protons in a direction transverse to the geomagnetic field lines, which has been observed on board the AUREOL 3 satellite, as shown in a companion paper. This process, which occurs only in multicomponent plasma, ultimately leads to the formation of a conical distribution, inside and above the heating region. It is shown that magnetosonic Alfven waves generated at higher altitudes can undergo a resonant mode conversion process as they propagate downward. The efficiency of the subsequent resonant absorption depends rather strongly on the propagation angle of the waves and on the relative abundance of $\mathrm{H}^{+}$ions: up to $25 \%$ of the incident energy flux can be transferred to protons. The present theory moreover foresees that a strong parallel electric field component has to occur at the altitude at which the wave frequency matches the proton gyrofrequency. The corresponding spectral signature has been unambiguously identified in the AUREOL 3 data, together with an intensification of the upward flux of suprathermal protons, which indicates the presence of proton conics. We have verified that within the uncertainties of the measurements the energy balance between the incoming electromagnetic power and the outgoing flux of kinetic energy is well explained by the theory.
\end{abstract}

\section{INTRODUCTION}

Since the first observations of ion "conics" by Sharp et al. [1977], much work has been done on this phenomenon, both observationally (see review by Klumpar [1986] or Burch [1988] and references therein) and theoretically. This interest is related to the fact that this process leads to the extraction of a large number of ions from the ionosphere. It is therefore an efficient way of supplying suprathermal ions into the magnetosphere.

It is widely admitted that the observed conical shape of the ion distribution function results from a two-stage process involving (1) an acceleration transverse to the magnetic field and (2) an upward motion along the geomagnetic field lines, under the effect of the mirror force. This second stage is easy to understand; the conservation of the first adiabatic invariant leads to a decrease of the semiapical angle of the conic as the ion moves upward from the acceleration region. The acceleration stage, however, still remains a challenging question. Many different heating or acceleration mechanisms have already been invoked. More than one could play a role in the formation of conics, taking into account the variety of waves generated in different magnetospheric regions.

Ungstrup et al. [1979] first suggested that electrostatic ion cyclotron waves (EICWs), driven unstable by a field-aligned current, accelerate the ions in a direction transverse to the magnetic field. This idea was further investigated, in partic-

\footnotetext{
${ }^{1}$ Entite Centre National de la Recherche Scientifique du Centre de Recherche en Physique de l'Environnement, Vélizy, France.

${ }^{2}$ Centre de Recherche en Physique de l'Environnement, Centre National d'Etude des Télécommunications, Centre National de la Recherche Scientifique, Issy-les-Moulineaux, France.

${ }^{3}$ Laboratoire de Physique et Chimie de l'Environnement, Centre National de la Recherche Scientifique, Orléans, France.

${ }^{4}$ Centre d'Etude Spatial des Rayonnements, Centre National de la Recherche Scientifique, Toulouse, France.

Copyright 1993 by the American Geophysical Union. $0148-0227 / 93 / 91 \mathrm{JA}-02186 \$ 05.00$
}

ular by Dusenbery and Lyons [1981] and by Ashour-Abdalla and Okuda [1984]. Chang and Coppi [1981] advocated that lower hybrid waves, destabilized by beams of precipitating auroral electrons, locally heat the ions. Crew and Chang [1985] have analytically determined the shape of the distribution function which would result from the resonant interaction between ions and lower hybrid waves, and Retterer et al. [1986] have numerically proven the efficiency of the overall process.

Although both EICWs and lower hybrid waves have been observed in various magnetospheric regions [Kintner et al., 1978; Mozer et al., 1979], these interpretations are at variance with some observations that do not establish a clear correspondence between such electrostatic waves and the occurrence of conics. This is the case of $\mathrm{O}^{+}$conics measured in the central plasma sheet on board the high-altitude polar-orbiting satellite DE 1 [Klumpar, 1986; Winningham and Burch, 1984]. What is generally observed, instead, is an intense flux of broadband ULF electromagnetic waves propagating downward through the magnetic flux tubes where conics are produced [Gurnett et al., 1984]. This led to the idea that the perpendicular heating of ions that leads to the formation of conics might be due to the gyroresonant interaction of minor ions (e.g., $\mathrm{O}^{+}$, at high altitudes) with these electromagnetic waves [Chang et al., 1986; Crew and Chang, 1988]. These authors have introduced the concept of ion cyclotron resonance heated conics (ICRHC) to explain the main features of the distribution function of the suprathermal $\mathrm{O}^{+}$observed by DE 1 [Crew et al., 1990]. In the ICRHC framework, as in other heating mechanisms, the ion motion essentially follows, to the lowest order, the adiabatic approximation. The perpendicular acceleration undergone by the ions at each altitude does not need to be very large, provided that the overall process is cumulative such that the energy of the conics will increase with altitude. Since the ions can interact gyroresonantly with the observed broadband ULF waves over a large fraction of the geomagnetic field line, the acceleration can indeed be cumulative [Chang et al., 1986; Retterer et al., 1987; Crew et al., 1990].

For this process to be efficient, the spectral power of the 
waves must be sufficiently large at frequencies close to the local gyrofrequency of the heated ions. Moreover, the waves must have a significant left-handed component in this frequency range, such that the resonant wave-particle interaction can occur. Yet Alfvén waves are not expected to have a left-handed polarized component at the ion gyrofrequencies [Stix, 1962; Smith and Brice, 1964]. One possibility is to assume that the resonant process occurs at a frequency $\omega=$ $\Omega_{\mathrm{O}^{+}}+k_{\|} v_{\|}$, for $\mathrm{O}^{+}$heating. If the Doppler shift is large enough, the ions can resonantly interact with left-handed (LH) polarized waves both below and above their gyrofrequency [Chang et al., 1986]. This, however, would imply some kind of preheating (or preaccelerating) process in order to produce the required Doppler shift. The possibility that the acceleration process is linked to nonlinear doublecyclotron absorption has also been advocated [Temerin and Roth, 1986; Ball, 1989].

For obliquely propagating Alfvén waves and in the presence of magnetic field gradients, the mode conversion of downgoing right-handed (RH) Alfvén waves into upgoing LH ion cyclotron waves (ICWs) is also possible, as pointed out by Retterer et al. [1987] and by Johnson et al. [1988, 1989], in the case of $\mathrm{O}^{+}$conics. When the inhomogeneous magnetospheric plasma contains two ionic species, new cutoff $(\|k\|=0)$ or reflection points $\left(k_{\|}=0\right)$ can occur, which allow upgoing ICWs to be produced from the downgoing Alfvén waves. The latter waves undergo a resonance $\left(k_{\|} \rightarrow \infty\right)$ at the local ion gyrofrequencies and thus can transversally heat ions, even if they are initially cold. This gives rise to the so-called resonant absorption process [Ginzburg, 1970]; the efficiency of this heating process can be estimated from the energy deficit of the transmission/ reflection budget [Budden, 1966]. Even if a fluidlike cold plasma model is chosen to describe the plasma, which thus does not explicitly exhibit the kinetic cyclotron absorption process, the resonant absorption can be taken into account if one handles correctly, with respect to all causality requirements, the singularities of the differential equation describing the full wave propagation [Ginzburg, 1970]. The mathematical method we have used here is based on a Laplace integral solution of the full wave propagation equation, which is approximated near the gyrofrequency layer at which the wave frequency matches the ionic gyrofrequency. It closely follows that employed earlier to determine the efficiency of the plasma heating by radio waves, within thermonuclear fusion devices [Fuchs et al., 1981; White et al., 1982; Cairns and Lashmore-Davies, 1983; LashmoreDavies et al., 1985].

The present paper is devoted to a detailed analytical study of both mode conversion and resonant absorption as they occur in an inhomogeneous magnetoplasma that mainly contains $\mathrm{O}^{+}$ions, together with a small amount of protons. The heating process then occurs in the vicinity of the proton gyrofrequency layer. We do not consider the heating of minor $\mathrm{O}^{+}$ions, which will be studied in a forthcoming paper.

This theoretical study has been triggered by the analysis of observations made on board the AUREOL 3 satellite. The corresponding data are extensively discussed in a companion paper by Rauch et al. [this issue], and here we only briefly summarize the main results of this study. AUREOL 3 was a mid-altitude orbiting satellite designed to study the upper ionosphere, at altitudes ranging from 400 to $2000 \mathrm{~km}$. In these regions the minor ion species is usually hydrogen, in contrast to the conditions that prevail at higher altitudes. AUREOL 3 mainly observed $\mathrm{H}^{+}$conics, essentially within the so-called light ion trough [Taylor and Walsh, 1972], where at AUREOL 3 altitudes the relative density of the protons is particularly low (10\% or less). Coincident with these proton conics, strong activity of electromagnetic ELF waves was observed. Their spectra exhibit some very peculiar features, the most unforeseen being the occurrence of very narrow ( $\Delta \omega / \omega$ of a few percent) and intense spectral lines just below the gyrofrequency of the protons. Such a narrow-banded feature is only seen on the parallel component of the electric field $\left(E_{z}\right)$, and it will be shown to give evidence of the mode conversion plus resonant absorption process. The theory indeed predicts that the $E_{z}$ component of the waves strongly diverges at the proton gyrofrequency layer. Moreover, the precise study of the wave spectrum in this very narrow spectral range shows that the ellipticity of the waves there unambiguously changes from $\mathrm{RH}$ to $\mathrm{LH}$, exhibiting further evidence of the mode conversion process. All these phenomena, observed over the entire altitude range covered by AUREOL 3, are closely connected with the occurrence of enhanced fluxes or primarily upgoing suprathermal hydrogen ions, which are strong in the high-altitude part of the orbit.

The paper is organized as follows. Section 2 is devoted to a presentation of the model and of the equations that determine the behavior of the various components of the electromagnetic field. The main properties of the dispersion equation in a homogeneous plasma are briefly discussed. A qualitative discussion of the role of the inhomogeneity is also presented, to investigate the effect of the relative density of $\mathrm{H}^{+}$ions. The propagation equations are solved in section 3 using an approximation valid near the altitude at which the wave frequency matches the proton gyrofrequency. The efficiency of the resonant absorption process is determined, and the behavior of the solution near the coupling region is discussed. In section 4, present theoretical results are compared with the data from AUREOL 3. The details of the mathematics are presented in an appendix.

\section{Basic Equations}

\subsection{Propagation Equations in Cold Inhomogeneous Plasma}

The only inhomogeneity considered here is the gradient of the magnetic field intensity along $\mathrm{O}_{2}$, the direction of the geomagnetic field lines, taken to be vertical. Let us consider a low-frequency wave $\left(\omega \ll \Omega_{e}\right.$, $\omega_{p e}$, the electronic gyrofrequency and plasma frequency, respectively) propagating through such an inhomogeneous magnetized cold plasma with an electron density $n_{e}$ and two ion species $\mathrm{O}^{+}$ and $\mathrm{H}^{+}$with relative densities $\varepsilon_{\mathrm{O}^{+}}=n_{\mathrm{O}^{+}} / n_{e}$ and $\varepsilon_{\mathrm{H}^{+}}=$ $n_{\mathrm{H}^{+}} / n_{e}\left(\varepsilon_{\mathrm{O}^{+}}+\varepsilon_{\mathrm{H}^{+}}=1\right)$. Fourier analysis is performed in the direction perpendicular to the magnetic field. After some straightforward calculations the following equations are obtained:

$$
\begin{gathered}
b_{x}=i \frac{d}{d \xi} E_{y} \\
b_{y}=-\frac{i}{1-\left(n_{\perp}^{2} / p\right)} \frac{d}{d \xi} E_{x}
\end{gathered}
$$




$$
\begin{gathered}
b_{z}=n_{\perp} E_{y} \\
E_{z}=\frac{i n_{\perp}}{p\left[1-\left(n_{\perp}^{2} / p\right)\right]} \frac{d}{d \xi} E_{x}
\end{gathered}
$$

and

$$
\begin{gathered}
\left(\frac{d^{2}}{d \xi^{2}}+s-n_{\perp}^{2}\right) E_{y}+i d E_{x}=0 \\
\frac{d}{d \xi}\left[\frac{1}{1-\left(n_{\perp}^{2} / p\right)} \frac{d}{d \xi} E_{x}\right]+s E_{x}-i d E_{y}=0
\end{gathered}
$$

Here $\xi=4 A(\omega / c) z$ and $b_{x, y, z}=(c / 4 A) B_{x, y, z}$, where $c$ is the velocity of light. $B_{x, y, z}$ and $E_{x, y, z}$ are the components of the magnetic and electric fields of the waves. The parameter $A=$ $\mu^{1 / 2} \omega_{p e} / \Omega_{e} \gg 1$ is here taken as a scaling constant, despite the variation of the magnetic field along $z$ (more precisely, the nondimensional variable $\xi$ can be thought of as divided by $\left.\int A d z\right) ; \mu$ is the ratio between the proton and the electron rest masses. The quantities $n_{\perp}, p, s=1 / 2(r+l)$, and $d=1 / 2(r-l)$ are derived from the perpendicular index of refraction $N_{\perp}=k_{\perp} c / \omega$ and from the usual functions of the cold magnetoionic theory $(P, S, D, R, L)[S t i x, 1962$, chapter 2] by the scaling transformation

$$
\left(n_{\perp}^{2}, p, s, d, r, l\right)=(4 A)^{-2}\left(N_{\perp}^{2}, P, S, D, R, L\right)
$$

Since the wave frequency is supposed to be small with respect to $\omega_{p e}$ and $\Omega_{e}$, these various quantities can be written as follows, to the lowest order in the small quantity $(4 A)^{-2}$ :

$$
\begin{gathered}
r=\frac{\varepsilon_{\mathrm{O}^{+}}}{16 x+1}+\frac{\varepsilon_{\mathrm{H}^{+}}}{16(x+1)} \\
l=-\left\{\frac{\varepsilon_{\mathrm{O}^{+}}}{16 x-1}+\frac{\varepsilon_{\mathrm{H}^{+}}}{16(x-1)}\right\} \\
s=-\left\{\frac{\varepsilon_{\mathrm{O}^{+}}}{(16 x)^{2}-1}+\frac{\varepsilon_{\mathrm{H}^{+}}}{16\left(x^{2}-1\right)}\right\} \\
d=x\left\{\frac{16 \varepsilon_{\mathrm{O}^{+}}}{(16 x)^{2}-1}+\frac{\varepsilon_{\mathrm{H}^{+}}}{16\left(x^{2}-1\right)}\right\} \\
p=-\frac{\mu}{16 x^{2}}
\end{gathered}
$$

We have introduced the normalized frequency $x(\xi)=$ $\omega / \Omega_{\mathrm{H}^{+}}, \Omega_{\mathrm{H}^{+}}$being the proton gyrofrequency. Equations $(1 b),(1 d)$, and $(2 b)$ can be further simplified by noting that in the frequency range under consideration, $\|p\|$ is a large number $\left(\geq 10^{2}\right)$ so that $n_{\perp}^{2} \leq 1 \ll\|p\|$.

\subsection{Dispersion Equation: Modes and Branches}

For a homogeneous plasma the dispersion equation and the mode polarization can be obtained from equations (1), (2), and (3) after a Fourier transform is performed on the spatial variable $\xi$. One then gets

$$
\mathscr{D}(n, x) \equiv\left(n^{2}-l\right)\left(n^{2}-r\right)+n_{\perp}^{2}\left(n^{2}-s\right)=0
$$

This biquadratic polynomial provides two solutions for the square of the parallel index of refraction, corresponding to two modes:

$$
n_{ \pm}^{2}=s-\frac{n_{\perp}^{2}}{2} \pm\left\{d^{2}+\frac{n_{\perp}^{4}}{4}\right\}^{1 / 2}
$$

Three branches correspond to these two modes (positive and negative). These branches are referred to as I, II, and III in the work by Rauch and Roux [1982] and Rauch et al. [this issue], and we shall here follow the same numbering. The topologies of the corresponding dispersion curves, which depend on $n_{\perp}$, are sketched in Figure 1. Notice, for further reference, that when one passes through a gyrofrequency, the divergence of the square root appearing on the right-hand side of (5) means that a given mode does not correspond to the same branch above and below this frequency. For this reason, a given branch can correspond to different modes, depending on the value of $x$ : II (III) corresponds to the positive mode for $x>x_{\mathrm{H}^{+}}\left(x>x_{\mathrm{O}^{+}}\right)$and to the negative mode for $x<x_{\mathrm{H}^{+}}\left(x<x_{\mathrm{O}^{+}}\right)$.

Besides the two ion gyrofrequencies $\left(x_{\mathrm{H}^{+}}=1, x_{\mathrm{O}^{+}}=\right.$ $1 / 16$ ), four characteristic real frequencies play an important role in determining the propagation and polarization properties of the propagating waves [Smith and Brice, 1964]. The reflection frequency

$$
x_{r}=\left\{\left(1-\frac{15}{16} \varepsilon_{\mathrm{H}^{+}}\right) \frac{1-(15 / 16) \varepsilon_{\mathrm{H}^{+}}-n_{\perp}^{2}}{1-n_{\perp}^{2}\left(1+15 \varepsilon_{\mathrm{H}^{+}}\right)}\right\}^{1 / 2}
$$

is the minimum frequency of the magnetosonic branch (II), for $n_{\perp}^{2}<\left(1+15 \varepsilon_{\mathbf{H}^{+}}\right)^{-1}$, or of the $\mathrm{H}^{+}$cyclotron branch (III), for $n_{\perp}^{2}>1-(15 / 16) \varepsilon_{\mathrm{H}^{+}}$. The minimum $x_{r}$ for branch II (for $n_{\perp}=0$ ) is the cutoff frequency $x_{c o}=1-(15 / 16) \varepsilon_{\mathrm{H}^{+}}$, and the maximum $x_{r}$ for branch III is the bi-ion frequency $x_{\text {bion }}=\left\{\left[1-(15 / 16) \varepsilon_{\mathrm{H}^{+}}\right] /\left[1+15 \varepsilon_{\mathrm{H}^{+}}\right]\right\}^{1 / 2}$ where this branch undergoes a resonance $\left(n_{\perp} \rightarrow \infty\right)$.

At the crossover frequency $x_{\text {crover }}=\left[1-(255 / 256) \varepsilon_{\mathrm{H}^{+}}\right]^{1 / 2}$ where the polarization of the propagating modes is linear, the $\mathrm{H}^{+}$cyclotron branch III and the magnetosonic branch II verify $n^{2}=1 / 17$ (fixed point of the dispersion curves) and $n^{2}+n_{\perp}^{2}=1 / 17$, respectively. The magnetosonic branch is RH for $x \geq x_{\text {crover }}$ and LH for $x \leq x_{\text {crover }}$. The converse is true for the $\mathrm{H}^{+}$cyclotron branch. The $\mathrm{O}^{+}$cyclotron branch (I) is always LH. The above quoted frequencies always verify the inequality (Figure 2 )

$$
1 / 16=x_{\mathrm{O}^{+}} \leq x_{\text {bion }} \leq x_{\text {co }} \leq x_{\text {crover }} \leq x_{\mathrm{H}^{+}}=1
$$

\subsection{Qualitative Discussion of the Coupling Process}

Prior to describing the mathematical formalism allowing us to study the coupling and absorption processes, a qualitative discussion might be helpful.

The heating of the $\mathrm{H}^{+}$ions results from the conversion of initially downgoing magnetosonic waves (MSW) into upgoing proton cyclotron waves $\left(\mathrm{H}^{+} \mathrm{ICW}\right)$. The latter waves have the possibility of reaching, "from below," the layer at which their frequency matches $\Omega_{\mathrm{H}^{+}}$(or $x=1$ ). As these latter waves are left handed, cyclotron absorption can occur, resulting in the perpendicular heating of the protons. Thus, before being absorbed, the electromagnetic energy thus has first to be reflected, which occurs essentially when the wave frequency matches the reflection frequency $x_{r}$ (in unit of 

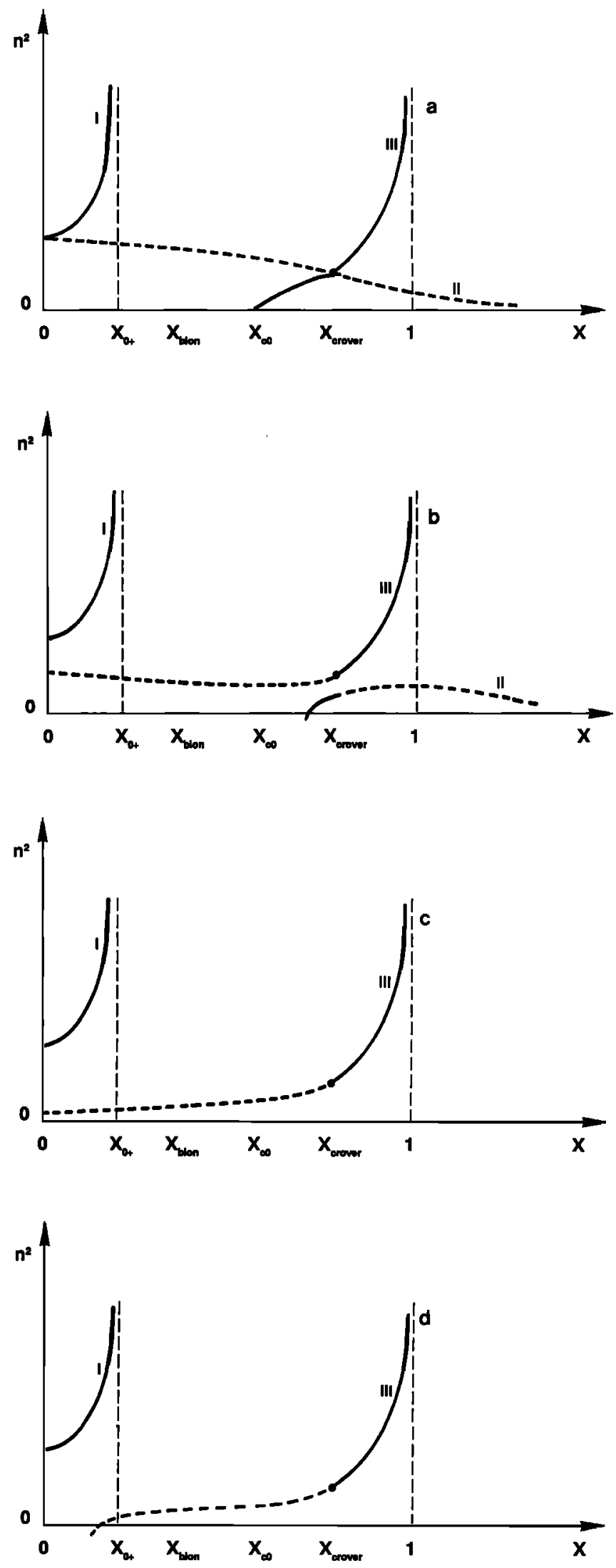

Fig. 1. Qualitative sketch of the shape of the dispersion curves for the various propagating modes of a magnetized plasma containing two ion species. The square of the parallel index is shown as a function of the normalized frequency $x=\omega / \Omega_{\mathrm{H}^{+}}$. The following values of the perpendicular refractive index $n_{\perp}$ are considered: (a) $n_{\perp}^{2}=0,(b) n_{\perp}^{2} \in\left[0,\left(1+15 \varepsilon_{\mathrm{H}^{+}}\right)^{-1}\right],(c) n_{\perp}^{2} \in\left[\left(1+15 \varepsilon_{\mathrm{H}^{+}}\right)^{-1}\right.$, $\left.1-(15 / 16) \varepsilon_{\mathrm{H}^{+}}\right]$, and $(d) n_{\perp}^{2}>1-(15 / 16) \varepsilon_{\mathrm{H}^{+}}$.

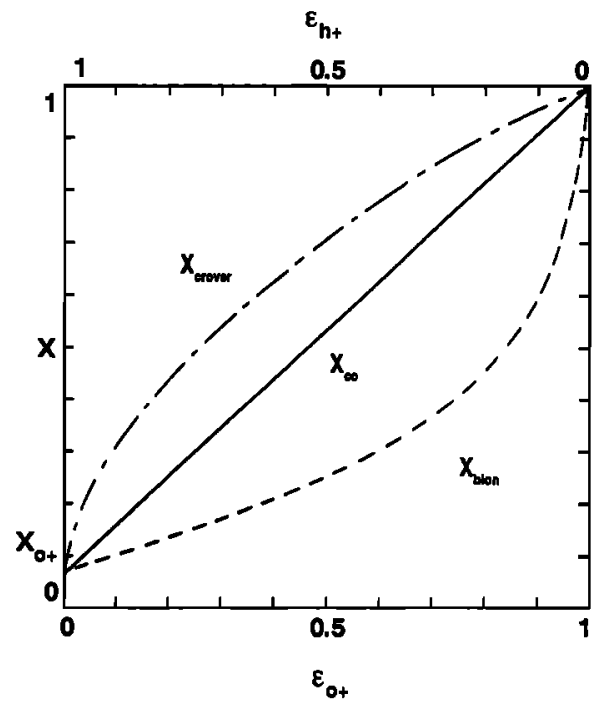

Fig. 2. Evolution of the various characteristic frequencies, $x_{\text {bion }}, x_{\mathrm{co}}$, and $x_{\text {crover }}$, as a function of the relative densities of the two ionic species $\mathrm{H}^{+}$and $\mathrm{O}^{+}$.

$\boldsymbol{\Omega}_{\mathrm{H}^{+}}$). One thus intuitively expects that the absorption process will be more efficient if this frequency lies below, but not far from, $\Omega_{\mathrm{H}^{+}}$. The proximity of the two frequencies will indeed allow the converted electromagnetic power to immediately reach the absorption layer without a complex intermediary propagation path. Moreover, the amplitude of the electromagnetic field is then expected to be strong because of the decrease of the group velocity occurring near the reflection layer. As shown by Fuchs et al. [1981] and more specifically, for the case we are dealing with here, by Lashmore-Davies et al. [1985], the coupling has to be studied in the complex frequency plane (and thus the complex $\xi$ plane). It is expected to occur at the points where the two solutions of the local dispersion equation, $\mathscr{D}[n, x(\xi)]=$ 0 , coincide, and thus at $\xi_{c}$, where $n_{+}\left(\xi_{c}\right)=n_{-}\left(\xi_{c}\right)$, or $n_{1}^{4}+4 d^{2}=0$. The point $\xi_{0}$, where the wave frequency matches the reflection frequency $x_{r}$ (corresponding to $n=$ 0 ), and $\xi_{c}$ are the branch points of the mapping $\xi \rightarrow n(\xi)$ in the complex plane. At both points the two conditions $\mathscr{D}(n, \xi)$ $=0$ and $\partial / \partial n \mathscr{D}(n, \xi)=0$ are indeed verified. When $\varepsilon_{\mathrm{H}^{+}} \ll$ 1 , it is simple to show that $x\left(\xi_{c}\right)$, the complex normalized frequency at which the coupling occurs, verifies

$$
x\left(\xi_{c}\right)=1-\frac{\varepsilon_{\mathrm{H}^{+}}}{2\left(1 \pm 8 \sin _{\perp}^{2}\right)}
$$

For $\varepsilon_{\mathrm{H}^{+}} \ll 1$ the real part of $x\left(\xi_{c}\right)$ remains near $\Omega_{\mathrm{H}^{+}}$for all the propagation angles of the incident Alfven waves, parameterized by $n_{\perp}$. Then the characteristic frequencies where wave reflection, mode conversion, and resonant absorption all occur are in the near vicinity of the proton gyrofrequency for a very broad range of $n_{\perp}$. Thus ion heating is intuitively expected to be favored under such conditions.

Another reasoning, proposed by Johnson et al. [1989], consists in assuming that the coupling does occur at $x_{\text {crover }}$ (Figure 2) and that the wave energy then simply tunnels up to the gyrofrequency layer, where it is absorbed. The two branches indeed have the same linear polarization at this frequency, but they do not have the same wave vector, and the reason for assuming that the coupling occurs there and thus for approximating the full wave equation around this 
point is far from being clear. Moreover, this approximation cancels the role played by the singularities of the full wave equation, which occurs at the gyrofrequencies of the ionic species and at which various components of the electromagnetic field of the solution exhibit a divergent behavior, which leads to observable effects, as shown by the AUREOL 3 data. To retain this behavior in the wave equation is also essential in order to handle correctly the gyroabsorption of the upgoing converted waves.

\section{Solution of the Wave Equations}

\subsection{Simplified Wave Equations}

To go beyond the qualitative reasoning presented in section 2 and to explicitly determine the absorption rate, one now solves the full wave equation $(2 a)$ and $(2 b)$, which will be done as in the work by White et al. [1982], by a Laplace integral method. In order to get, from $(2 a)$ and $(2 b)$, equations amenable to an analytical solution, the expressions of the various functions $s, d, r, l$ are simplified such that the corresponding dispersion equation retains all the mathematical features which have been discussed in section 2. Since the singularity occurring at the proton gyrofrequency layer is essential for determining the behavior of the solution, we have chosen to approximate these functions by expressions which are fully valid in this range of altitudes. This moreover allows us to correctly handle the entire conversion-reflection-absorption process, which is expected to be favored in this vicinity.

It is assumed that $\varepsilon_{\mathbf{H}^{+}} \ll 1$, and only the linearized variation of the magnetic field amplitude occurring near the gyrofrequency layer is retained; one gets from $(2 a)$ and $(2 b)$

$$
\begin{array}{r}
\left(\frac{d^{2}}{d \xi^{2}}-\alpha-\frac{\eta_{\mathrm{H}^{+}}}{\xi}\right) E_{x}=i\left(\beta+\frac{\eta_{\mathrm{H}^{+}}}{\xi}\right) E_{y} \\
\left(\frac{d^{2}}{d \xi^{2}}-\alpha-n_{\perp}^{2}-\frac{\eta_{\mathrm{H}^{+}}}{\xi}\right) E_{y}=-i\left(\beta+\frac{\eta_{\mathrm{H}^{+}}}{\xi}\right) E_{x}
\end{array}
$$

with $\xi=4 A(\omega / c) L_{b}(x-1)$ and $\eta_{\mathrm{H}^{+}}=\varepsilon_{\mathrm{H}^{+}}\left(\omega L_{b} / 8 c\right) A$. Here $L_{b} \approx R / 3$ is the scale length of the magnetic gradient, $R$ the distance from the Earth's center to the coupling layer. The parameter $\eta_{\mathrm{H}^{+}}$is of the order of $\varepsilon_{\mathrm{H}^{+}}$times the product $L_{b} k$, where $k$ is the typical value of the MSW wave number, at the proton gyrofrequency: $\eta_{\mathbf{H}^{+}}$is thus a scaling parameter which, in some sense, determines the width of the coupling region measured with the dimensionless variable $\xi$. In these equations the parameters $\alpha$ and $\beta$ depend on $\varepsilon_{\mathrm{H}^{+}}$. To the lowest order, $\alpha=1 / 255$, and $\beta=16 / 255$. The role played by higher-order corrections in $\varepsilon_{\mathrm{H}^{+}}$will be discussed in the appendix (see formula (A5)). In particular, it can be shown that the coupling point loci linked to the local dispersion equation obtained from $\left(2 a^{\prime}\right)$ and $\left(2 b^{\prime}\right)$ are quantitatively similar to those which can be determined from the complex dispersion equation, for $\varepsilon_{\mathbf{H}^{+}}<0.9$, thus ensuring that the overall coupling process will be similar, in that parameter range.

Equations ( $\left.2 a^{\prime}\right)$ and $\left(2 b^{\prime}\right)$ describe the coupling between the right-handed MSW (II) and the left-handed $\mathrm{H}^{+}$EICW (III), which at infinity $(\xi \rightarrow \pm \infty)$ corresponds to the single propagating mode $n_{+}(\infty), n_{-}(\infty)$ being always purely imaginary (see Figure 3 ). The incidence angle of the waves with respect to the magnetic field lines, taken for $\xi \rightarrow \pm \infty$, far
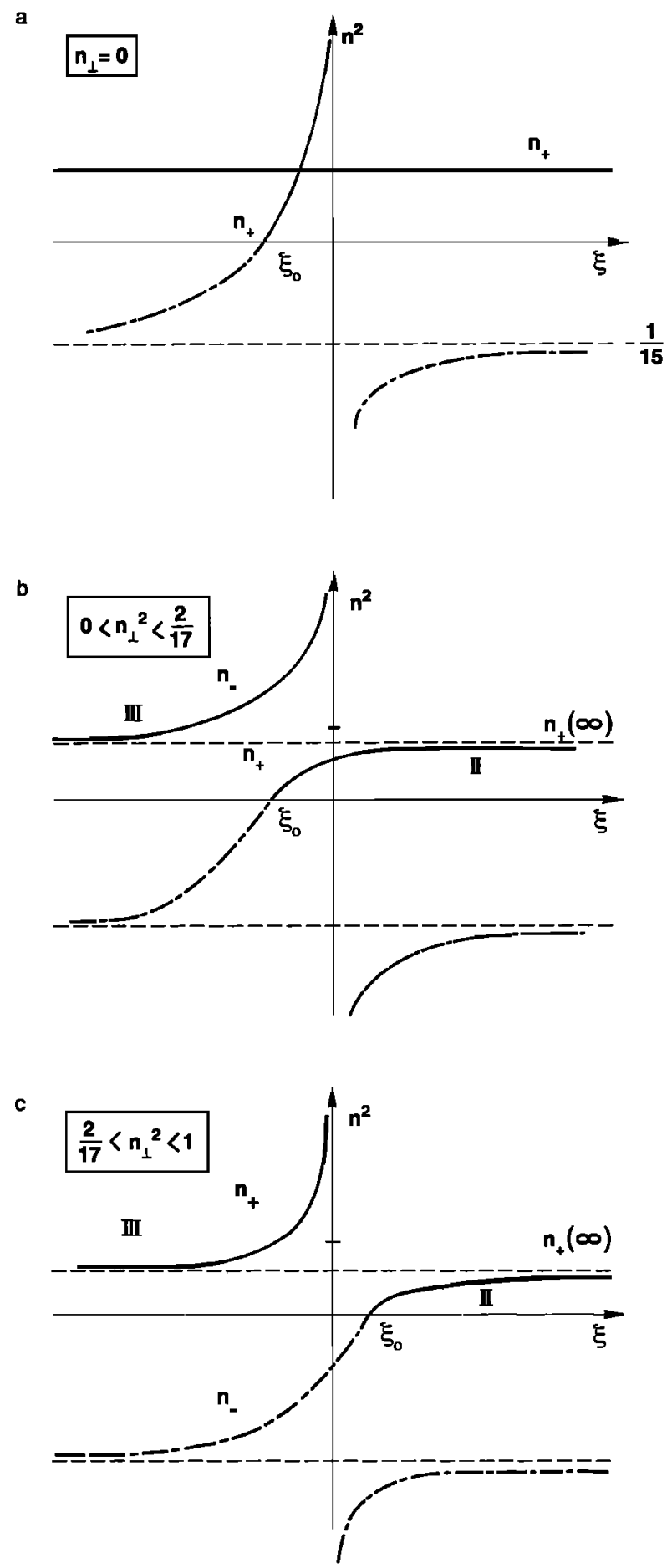

Fig. 3. The shapes of the dispersion curves near $\Omega_{\mathrm{H}^{+}}$, when $\varepsilon_{\mathrm{H}^{+}} \ll 1$. At $n_{\perp}^{2}=2 / 17$ the "classical" cutoff frequency of the MSW (type II branch) crosses $\Omega_{\mathrm{H}^{+}}$.

from the coupling point, is given by $\theta_{+}=\tan ^{-1}\left[n_{\perp} / n_{+}(\infty)\right]$. This corresponds to the propagation angle of the MSW linked to this mode, which has the approximate dispersion equation $\omega=k V_{A}$ (for $\Omega_{\mathrm{H}^{+}} \ll \omega \ll \Omega_{c e}$ ) where $V_{A}$ is the Alfvén velocity: once the frequency and the perpendicular wave vector are fixed (by the constant $n_{\perp}$ ), the angle of propagation is indeed determined. In what follows we will simply write $n_{ \pm}$for $n_{ \pm}(\infty)$.

In order to solve $\left(2 a^{\prime}\right)$ and $\left(2 b^{\prime}\right)$, we closely follow the Laplace integral method used by White et al. [1982]. The 
calculations are performed in the appendix. As a result, the various components of the electromagnetic field of the wave can be derived by applying simple differential operators to the function

$$
\begin{aligned}
& \Psi(\xi, \mathscr{C}) \\
& =\int_{\mathscr{C}} \frac{\exp (n \xi) d n}{\left(n+n_{+}\right)^{1+i \delta_{+}}\left(n+n_{-}\right)^{1+i \delta_{-}}\left(n-n_{-}\right)^{1-i \delta_{-}}\left(n-n_{+}\right)^{1-i \delta_{+}}}
\end{aligned}
$$

One gets

$$
\begin{gathered}
E^{-}=\frac{n_{\perp}^{2}}{2} \Psi(\xi, \mathscr{b}) \\
E^{+}=\left(\frac{n_{\perp}^{2}}{2}-\frac{1}{17}-\frac{d^{2}}{d \xi^{2}}\right) \Psi(\xi, \mathscr{b}) \\
E_{z}=\Gamma\left(\mu^{-1}\right) \frac{n_{\perp}}{2}\left[\left(n_{\perp}^{2}-\frac{1}{17}\right) \frac{d}{d \xi}-\frac{d^{3}}{d \xi^{3}}\right] \Psi(\xi, \mathscr{b})
\end{gathered}
$$

where $\Gamma=16$.

The $\delta_{ \pm}$are functions of $n_{\perp}$; they are determined in the appendix. $\mathscr{C}$ is an appropriate contour which is defined in accordance with the boundary conditions, namely that no power flux comes from below (from $\xi=-\infty$ ), to be consistent with a source located at high altitudes. The method used for choosing a contour that respects the causality conditions and the transformations which have to be applied to it, to ensure that the same convergent solution of the differential equations $\left(2 a^{\prime}\right)$ and $\left(2 b^{\prime}\right)$ is properly matched over the whole $\xi$ axis, is explained in the appendix. These matching conditions provide the transmission, reflection, and absorption factors corresponding to the overall coupling process. Moreover, once $\Psi$ is determined, equations $(8 a)-(8 c)$ allow us to estimate the spatial distribution of the components of the electromagnetic field, or their spectrum, as measured at a given altitude.

\subsection{Structure of the Wave Electric Field}

Since $n_{-}$is purely imaginary, only $n_{+}$intervenes in the coupling occurring near the proton gyrofrequency (the monomials linked to $n_{-}$in (7) do not vary much over the integration path). The contributions of that part of the contour $\mathscr{C}$ that encircles these purely imaginary poles can be neglected in (7). As discussed in the appendix, $\Psi$ can be expressed in terms of the so-called irregular Kummer's functions $U\left( \pm \delta_{+}, 0, \pm 2 n_{+} i \xi\right)$, which are "logarithmic" solutions of the confluent hypergeometric differential equation [Abramowitz and Stegun, 1970, chapter 13]; $\delta_{+}$is given by

$$
\delta_{+}=\eta_{H^{+}} \frac{\Delta^{1 / 2}-(16 / 255)}{2(\Delta)^{1 / 2}\left[\Delta^{1 / 2}-(1 / 255)-\left(n_{\perp}^{2} / 2\right)\right]^{1 / 2}}
$$

where $\Delta=n_{\perp}^{4} / 4+(16 / 255)^{2}$.

For $z \approx 0$ [Abramowitz and Stegun, 1970, chapter 13.5.11],

$$
U\left( \pm i \delta_{+}, 0, z\right) \approx 1 / \Gamma\left(1 \pm i \delta_{+}\right)+O(|z \ln z|)
$$

Thus, at $\xi=0, \Psi$ is finite but has a logarithmically divergent derivative. From (8) one sees that while the RH component of the electric field remains bounded at the gyrofrequency, the LH component diverges like $\xi^{-1}$, and the component $E_{z}$, parallel to the geomagnetic field line, diverges like $\xi^{-2}$. As seen by an observer measuring the electric field power spectrum near the conversion layer, this leads to a strong intensification of the parallel electric field at or near the proton gyrofrequency. The LH electric field component is also intensified, as expected for a conversion from RH into LH waves, but its effective divergence is smoother than that of the parallel component. Since no strong parallel electric field is expected to be observed otherwise in this frequency range, the measurement of this spectral peak is a major token of the role played by the inhomogeneity of the medium and thus strongly suggests the occurrence of the mode conversion process.

The cold plasma theory used in this study does not allow the determination of the width of this spectral peak. This is linked to the fact that this plasma model does not include any typical length (or time) for characterizing the resonant cyclotron wave-particle interaction which is at work during the absorption. Here this kinetic effect indeed only enters the equation through the $\xi^{-1}$ term appearing in $\left(2 a^{\prime}\right)$ and $\left(2 b^{\prime}\right)$, which comes from the divergent $\left(\omega-\Omega_{\mathrm{H}^{+}}\right)^{-1}$ cyclotron resonant terms occurring in the cold plasma dielectric tensor. This term allows the resonant process to occur only at a single point and gives rise to the divergent behavior of the solution. Conversely, if the thermal dispersion of the protons is taken into account, together with the magnetic field inhomogeneity, a characteristic interaction length $\Delta z$ can be constructed which gives a finite size to the coupling region, and thus a finite width to the spectral peak. Without going into the details of this calculation, which is left for a further work, an order-of-magnitude estimate can be given, for the sake of comparison with observations. In the spatial Fourier space the spatial width of the peak $\Delta z$ corresponds to a range of parallel wave vectors, $\Delta k_{\|} \approx 2 \pi / \Delta z$, and thus to a range of Doppler-shifted frequency $\Delta \omega \approx \Delta k_{\|}\left(v_{s h i}\right)_{\|}$as seen in the particle rest frame, $\left(v_{t h i}\right)_{\|}$being the parallel thermal velocity of the ions. On the other hand, this frequency range must also correspond to the frequency mismatch due to the detuning of the cyclotron resonance by the inhomogeneity of the magnetic field, over the interaction length $\Delta z: \Delta \omega \approx$ $\Omega_{\mathrm{H}^{+}}\left(\Delta z / L_{b}\right)$. Combining these two relations, one gets, for the minimal half width of the spectral peak,

$$
\Delta \omega=\left(2 \pi \frac{\Omega_{\mathrm{H}^{+}}\left(v_{t h i}\right)_{\|}}{L_{b}}\right)^{1 / 2}
$$

$(\Delta \omega)^{-1}$ can also be considered as a measure of the typical heating time, at a given altitude.

\subsection{Transmission, Reflection and Absorption}

As shown in the appendix, the energy transmission, reflection, and absorption factors of the earthward propagating magnetosonic waves are related in the usual way [Budden, 1966]:

$$
T=\exp \left(-2 \pi \delta_{+}\right) \quad R=(1-T)^{2} \quad A=T(1-T)
$$




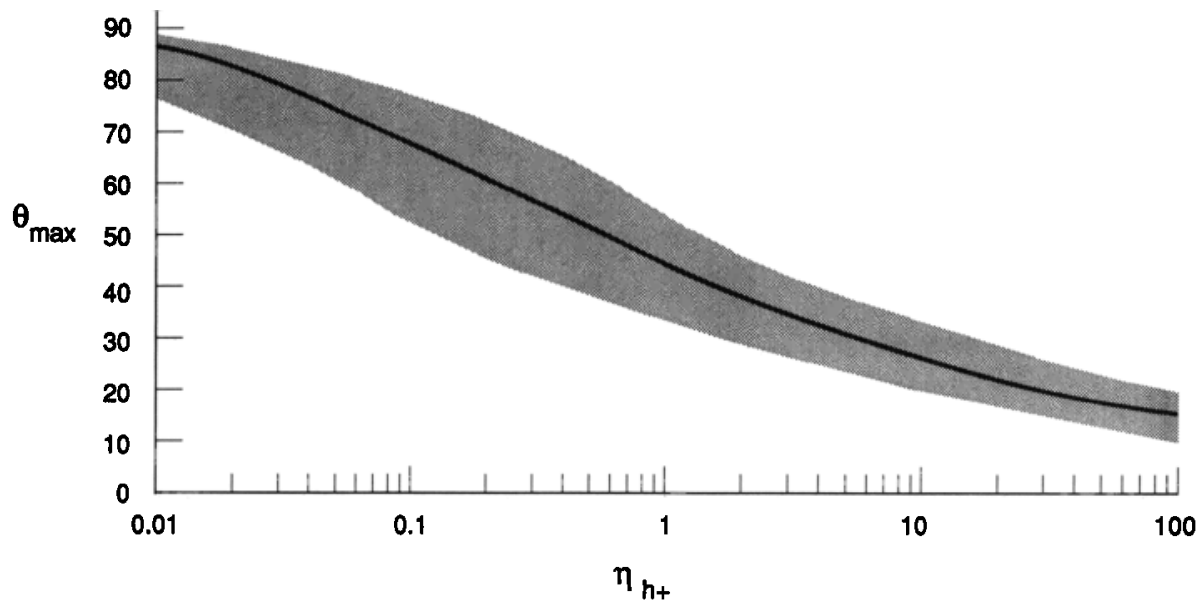

Fig. 4. Incidence angle of MSW for which the resonant absorption is maximum, as a function of the parameter $\eta_{\mathrm{H}^{+}}$. The shaded area corresponds to the half width of the absorption curve.

This is due to the fact that only a single resonance-cutoff pair plays a role, because only one mode propagates at infinity. The relation (11) between $R, A$, and $T$ can be interpreted in a very simple way, following a heuristic reasoning suggested by Cairns and Lashmore-Davies [1983]. Let us suppose that the electromagnetic energy initially comes from the right (e.g., from high altitudes) toward the conversion layer, on branch II (Figure 3). Let $T$ be the energy transmission coefficient from branch II to branch III, and thus on the same mode (also from branch III to branch II). Then, only a fraction $(1-T)$ of the incident power will be reflected, on branch II, near the reflection point $\xi_{0}$. Of this reflected power, a fraction $T$ will be again transferred to branch III and thus be incident from the left at the gyrofrequency level, where $\mathrm{H}^{+}$heating has to occur. The fraction of absorbed power will thus be $T(1-T)$. The remaining fraction of the incident power, $(T-1)^{2}$, will finally be reflected back from the conversion layer to the right.

As $n_{\perp}$ varies from 0 to 1 , the incident angle $\theta_{+}$varies from $0^{\circ}$ to $90^{\circ}$, and $\delta_{+}$, which has nothing to do with any "tunneling distance," varies from 0 to $\infty$. Thus the transmission coefficient $T=\exp \left(-2 \pi \delta_{+}\right)$varies from 1 to 0 , and the absorption rate is maximum for $A=0.25(T=0.5$, or $\delta_{+} \approx 0.11$ ) and thus for an intermediate angle which depends on $\eta_{\mathrm{H}^{+}}$(Figure 4). This will not necessarily correspond to a reflection point occurring below the $\mathrm{H}^{+}$gyrofrequency layer, which points out the weakness of the "intuitive" reasoning at the beginning of section 2.3.

The efficiency of the heating process will depend on the angular width of the absorption curve and on the distribution of the angles of incidence of the MSW. As shown in Figures 4 and 5 , the most efficient cases likely correspond to values of $\eta_{\mathrm{H}^{+}}$varying from 0.1 to 1 , when the absorption is strong over a large angular range. When $\eta_{\mathbf{H}^{+}}$is larger, absorption occurs only for small propagation angle and over a smaller angular width. When it is smaller, strong absorption is mainly restricted to incident angles very close to $90^{\circ}$.

Figure 6 shows how these results will be modified if finite- $\varepsilon_{\mathbf{H}^{+}}$corrections were retained in equations $\left(2 a^{\prime}\right)$ and $(2 b$ and thus in the expressions of $n_{+}$and $\delta_{+}$given in the appendix. It exhibits the evolution of the absorption curve for increasing values of $\varepsilon_{\mathrm{H}^{+}}$when the parameter $\eta_{\mathbf{H}^{+}}$is artificially kept constant. Then, the angle where the maximal absorption oc- curs decreases only slightly with $\varepsilon_{\mathrm{H}^{+}}$, as the half width of the absorption curve. The main variation of the absorption is thus given by the change of $\eta_{H^{+}}$. This is the optimizing value of this parameter, linked to both $\varepsilon_{\mathrm{H}^{+}}$and the typical magnetic gradient length $L_{b}$ (always of the order of the Earth's radius), which makes it necessary for the hydrogen to be a minor species in order to be efficiently heated.

\section{Discussion}

On board the AUREOL/ARCAD 3 satellite, five components of the electromagnetic field (two electric, three magnetic) were measured in waveforms. Rauch et al. [this issue] give evidence of the signature of resonant absorption events observed at altitudes ranging from 1000 to $2000 \mathrm{~km}$, in the light ion trough (LIT). During such events the dynamic spectrum of the magnetic component shows a low-frequency cutoff, slightly below the proton gyrofrequency $f_{\mathrm{H}^{+}}$(typically $5-10 \mathrm{~Hz}$ below). The polarization of the electromagnetic turbulence, which was RH above $f_{\mathrm{H}^{+}}$, becomes linear just below and LH close to the cutoff frequency $f_{\text {co }}$. This could a priori be interpreted as a simple polarization reversal at the crossover frequency $f_{\text {crover, }}$, which would explain why a downgoing type II MSW would change from $\mathrm{RH}$ to $\mathrm{LH}$. Electric field data, however, cannot be explained only by a polarization reversal, for the following reasons. First, the transverse component of the electric field $\left(E_{H}\right)$ decreases just below $f_{H^{+}}$, before falling to noise level below $f_{\text {co }}$. Such a decrease of $E H$ is not consistent with a simple reflection of MSWs at $f_{\text {co }}$, which would only give an enhancement of the spectral power, at this frequency, due to an Airy function effect. Second, the parallel component $\left(E_{z}\right)$ shows a large peak between $f_{\text {co }}$ and $f_{\mathrm{H}^{+}}$. If the observed waves were MSWs, $E_{z}$ would be exceedingly small. Thus, below $f_{\mathrm{H}^{+}}$, at least part of the energy of the downgoing MSWs has to mode convert into a type III $\mathrm{H}^{+} \mathrm{ICW}$. Present calculations support this idea. Indeed, in section 3 the parallel electric field resulting from the resonant mode conversion process has been shown to be large in the conversion layer, and even to diverge at $f_{\mathrm{H}^{+}}$. The $E_{H}$ component is still expected to have large values, but its divergence is still smoother, and the expected very small value of $E_{z}$, except at $f_{\mathrm{H}^{+}}$, must produce the very large contrasting effect which is indeed 


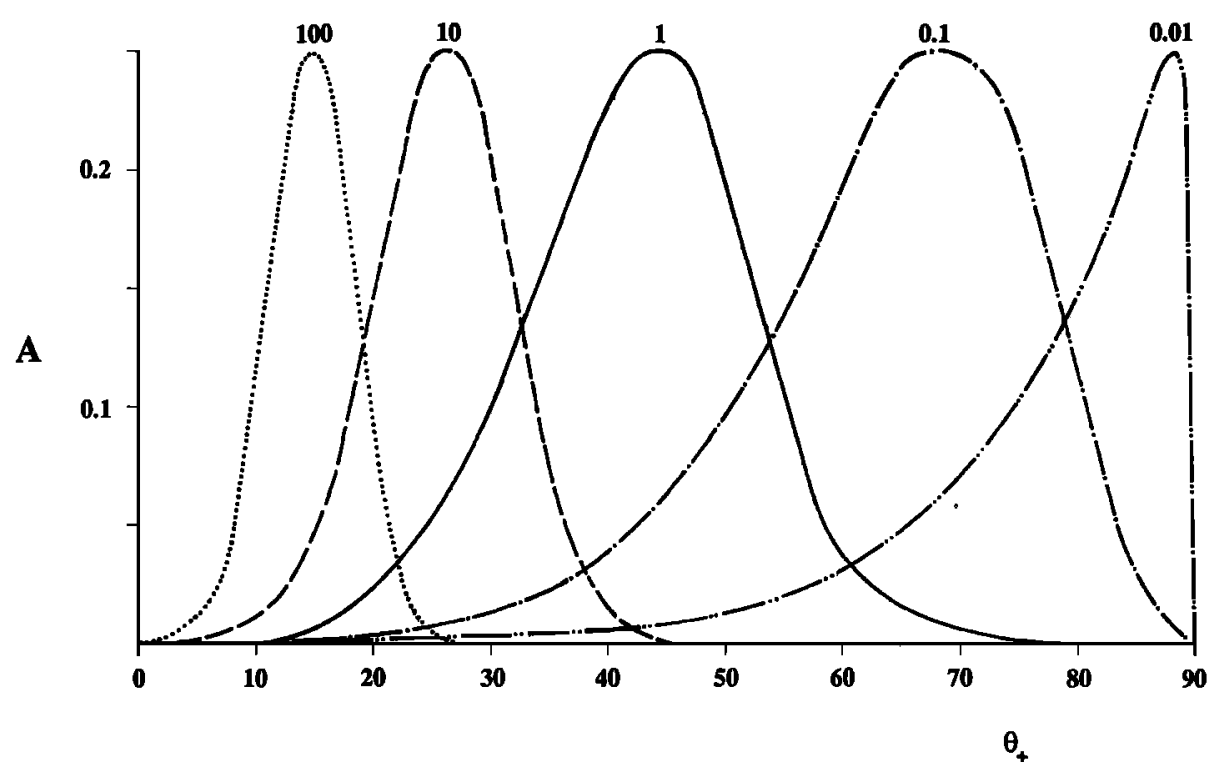

Fig. 5. Values of the absorption coefficient as a function of $\theta_{+}$, the incidence angle of the MSW far from the gyrofrequency layer. Five values of $\eta_{\mathrm{H}^{+}}$have been used, from $\eta_{\mathrm{H}^{+}}=10^{2}$ (left curve) to $\eta_{\mathrm{H}^{+}}=10^{-2}$ (right curve); $\eta_{\mathrm{H}^{+}} \approx 10$ is relevant to the event of orbit 3162 , studied by Rauch et al. [this issue].

observed. While not important for the heating of the $\mathrm{H}^{+}$ions by itself, the intense and narrow spectrum of $E_{z}$ gives evidence of the existence of a resonant mode conversion of the downgoing MSW into an upgoing ICW, close to $f_{\mathrm{H}^{+}}$. The heating of the $\mathrm{H}^{+}$ions is due to the transverse component $\left(E_{H}\right)$ of the electric field, which is consistent with the observed decrease of $E_{H}$ between $f_{\text {co }}$ and $\mathrm{H}^{+}$. The abrupt polarization change just below $f_{\mathrm{H}^{+}}$is also consistent with a resonant mode conversion and absorption process; indeed the theory foresees that the ratio $\left\|E^{+}\right\| /\left\|E^{-}\right\|$diverges at the same location as the $E_{z}$ component.

We expect the slight frequency difference between the peak in $E_{z}$ and $f_{\mathrm{H}^{+}}(\approx 5 \mathrm{~Hz})$ to be linked to thermal effects, which also have to explain the observed spectral width of the peak. For the event of orbit 3162, described by Rauch et al. [this issue], the magnetic gradient length can be estimated to be $L_{b} \approx 2.5 \times 10^{6} \mathrm{~m}$, and the proton gyrofrequency $f_{\mathrm{H}^{+}}=$ $(2 \pi)^{-1} \Omega_{\mathrm{H}^{+}} \approx 400 \mathrm{~Hz}$. Assuming $1 / 2 m\left(v_{\text {thi }}\right)^{2} \| 10 \mathrm{eV}$, one gets from equation (10) $\Delta f \approx 2.2 \mathrm{~Hz}$. This is indeed of the order of magnitude of the half width of the observed spectral peak.

The efficiency of the energy absorption process depends only upon the size of the positive parameter $\eta_{\mathrm{H}^{+}}$, and thus the value of both $\varepsilon_{\mathrm{H}^{+}}$and the angular range covered by the downgoing MSW. From the measured frequency cutoff of the MSW, $\varepsilon_{\mathrm{H}^{+}} \approx 10 \%$ for orbit 3162 [Rauch et al., this issue]. With the plasma parameters corresponding to the studied event, one gets $\eta_{\mathrm{H}^{+}} \approx 10$. As shown in Figures 4, 5 , and 6 , the heating process thus maximizes for $\theta_{+} \approx 20^{\circ}-25^{\circ}$, which is consistent with the propagation angle of the incident

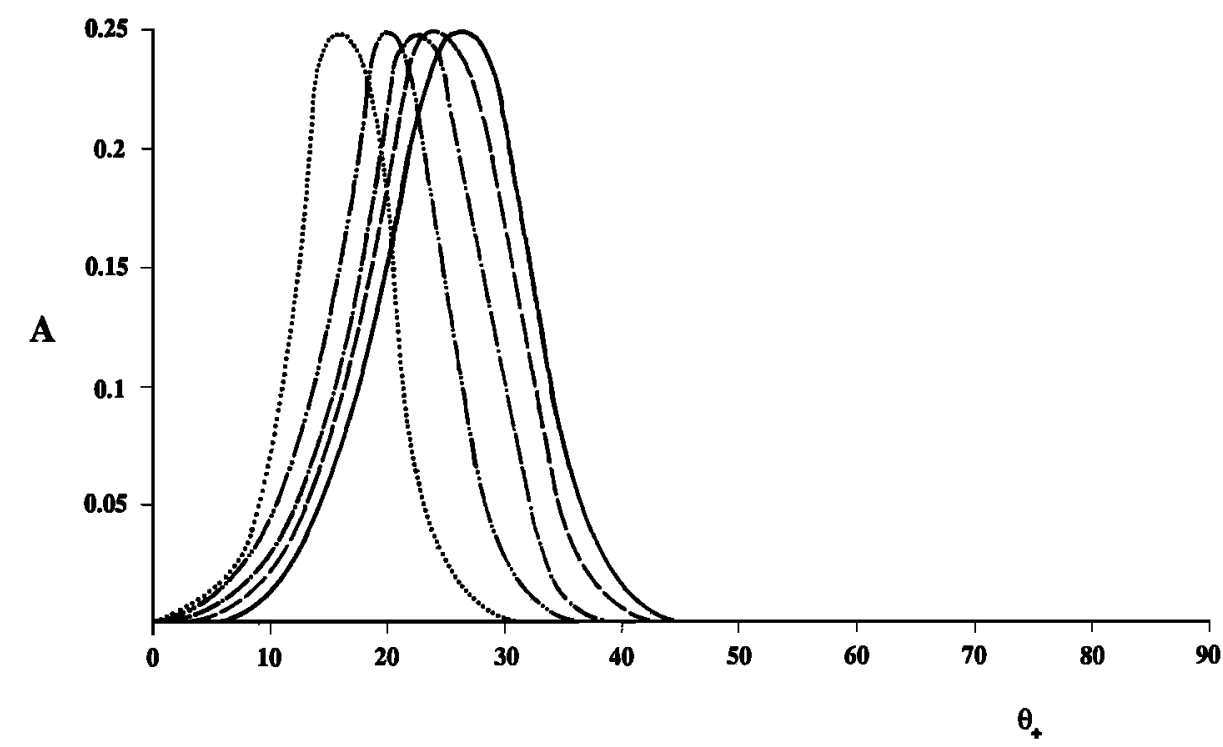

Fig. 6. Same as Figure 5, but $\eta_{\mathrm{H}^{+}} \approx 10$ is artificially maintained constant and $\varepsilon_{\mathrm{H}^{+}}$is varied to show the effect of the finite $-\varepsilon_{\mathrm{H}^{+}}$corrective terms: $\varepsilon_{\mathrm{H}^{+}}=0$ (solid), 0.25 (dashed), 0.5 (dot-dashed), 0.75 (dot-dot-dashed), and 1 (dotted). 
Alfvén waves which has been determined from the observations [Rauch et al., this issue].

According to the present calculation, the parallel phase velocity of the downgoing MSWs must be of the order of

$$
V_{\phi \|} \approx\left[c / n_{+}(\infty)\right]\left(\Omega_{e} / \omega_{p e}\right) /(16 \mu)^{1 / 2} \approx c / 40
$$

with $n_{+}(\infty)=(17)^{-1 / 2}$ and $\Omega_{e} \approx \omega_{p e}$, as observed during the event of orbit 3162 . This value of the phase velocity can be checked from the data by determining the experimental value of the ratio $\delta E_{H} / \delta B_{H} \approx 10^{-7}$ in this frequency range.

Particle data give evidence of enhanced fluxes of suprathermal protons, with an upward flux larger than the downward one, during the same event. We interpret this asymmetry in the observed proton fluxes as the signature of a proton conic resulting from the cumulative acceleration of hydrogen ions below the satellite. Since the altitude is relatively large for the quoted event $(\approx 1950 \mathrm{~km})$, the acceleration of the ions is expected to take place over a broad altitude range below the satellite $(\Delta h \approx 1000 \mathrm{~km})$, thus leading to the formation of a hydrogen ICRHC, which is here observed as a strong enhancement of the flux of suprathermal upgoing protons.

Let us now compare the expected heating with the flux of kinetic energy emerging from the postulated heating region. One has first to determine the electromagnetic energy input. For orbit 3162 the typical values of the spectral densities, for each component of the electromagnetic field, are $\delta B^{2} \approx$ $2.5 \times 10^{-5} \gamma^{2} \mathrm{~Hz}^{-1}$ and $\delta E^{2} \approx 4 \times 10^{-3}(\mathrm{mV})^{2} \mathrm{~Hz}^{-1}$. The approximate energy flux entering the box where the wave absorption by the observed protons has to occur thus is written

$$
\Phi_{e m} \approx \frac{V_{g \|} \Delta f}{4 \mu_{0}}\left\{3 \delta B^{2}+2\left(\frac{\delta E}{c}\right)^{2}\right\}
$$

where $V_{g \|}$ is the parallel group velocity of the incident waves over the frequency range $\Delta f=3 \Delta h / R f_{\mathrm{H}^{+}} \approx 200 \mathrm{~Hz}$ above the local proton gyrofrequency, which is the range of proton gyrofrequencies over the altitude range $\Delta h \approx 1000$ $\mathrm{km}$. In (13) the factors 3 and 2 correspond to the number of field components of the propagating waves. Slightly above the proton gyrofrequency, $V_{g \|}$ is expected to be of the order of $V_{\phi \|} \approx c / 40$. In fact, the parallel group velocity of the magnetosonic waves has a nontrivial behavior, as a function of both $n_{\perp}$ and the frequency $\left(x=\omega / \omega_{\mathrm{H}^{+}}\right)$. In Figure 7 the shaded area gives the value of $V_{g \|}$ for the range of asymptotic angles of propagation corresponding to the Gaussian shaped absorption curve of Figure 5, with $\eta_{\mathrm{H}^{+}} \approx 10$. For the frequency range of interest (from $x \approx 1$ to $x \approx 2$ ), one thus gets $V_{g \|} \approx c / 20$ as a mean value. From the data and equation (13) the contribution of the electric field is clearly negligible in the determination of the energy flux, and one finally gets the incident electromagnetic flux:

$$
\Phi_{e m} \approx 9 \times 10^{-8} \mathrm{~J} \mathrm{~m}^{-2} \mathrm{~s}^{-1}
$$

Taking into account that only one quarter of this energy can be converted into mechanical energy, one gets an available energy flux of $\approx 2.2 \times 10^{-8} \mathrm{~J} \mathrm{~m}^{-2} \mathrm{~s}^{-1}$ for heating the protons below the satellite.

The output of mechanical energy can be roughly determined from the particle data presented in the companion paper. The net $\mathrm{H}^{+}$energy flux $\Phi_{\mathrm{H}^{+}}$is given by

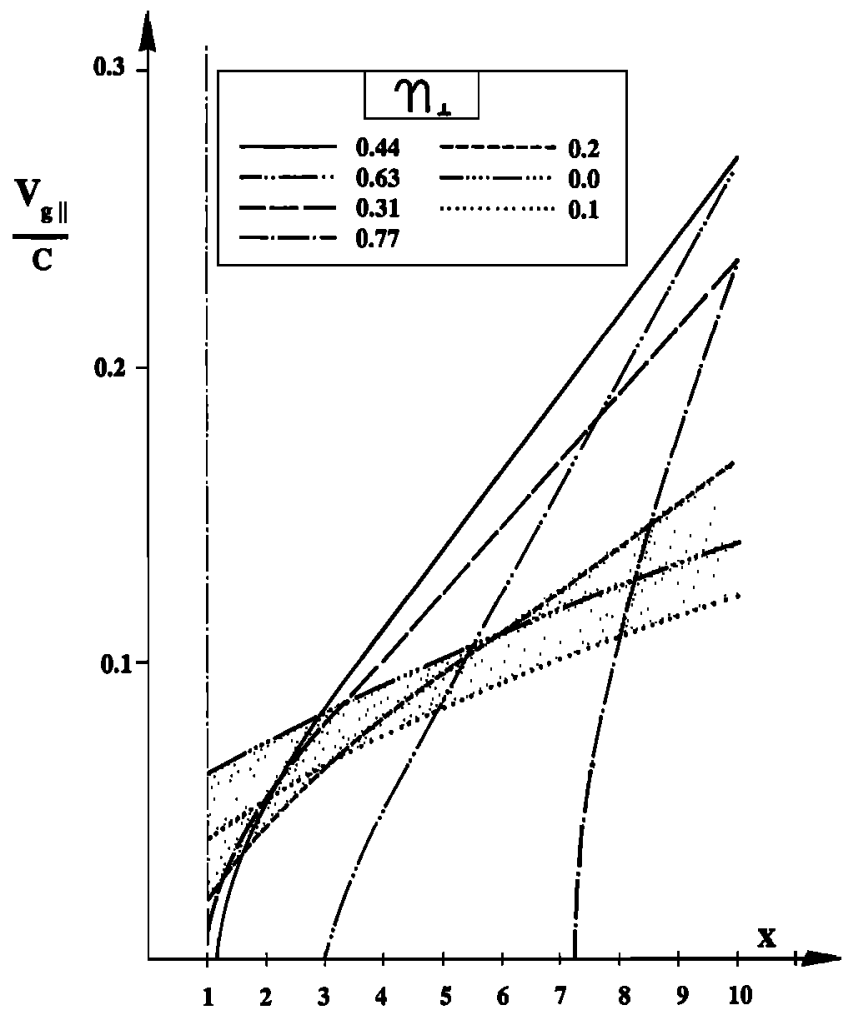

Fig. 7. The group velocity of the magnetosonic waves, as a function of the normalized frequency $x=\omega / \Omega_{\mathrm{H}^{+}}$. Here $\eta_{\mathrm{H}^{+}}=10$, as for the event of orbit 3162 . Each curve corresponds to a value of $n_{\perp}$, given in the figure. The shaded area corresponds to the values of the parameter that lead to a strong absorption coefficient, as shown in Figure 5.

$$
\Phi_{\mathrm{H}^{+}}=2 \pi \int_{0}^{\pi} \sin (\alpha) d \alpha \int_{0}^{\Delta E_{m}} j(\alpha, E) E d E
$$

where $j(\alpha, E)$ is the differential flux at energy $E$ and pitch angle $\alpha$. If we assume that downward and upward ion fluxes remain isotropic and constant within the lowest energy channel of width $\Delta E_{m} \approx 100 \mathrm{eV}, \Phi_{\mathrm{H}^{+}}$can be approximated by

$$
\begin{aligned}
\Phi_{\mathrm{H}^{+}} \approx \pi \Delta E_{m}^{2}\left[j_{\downarrow}\right. & \left.-\cos \alpha_{0} j_{\uparrow}\right] \\
& \approx 5 \times 10^{-14}\left[j_{\downarrow}-\cos \alpha_{0} j_{\uparrow}\right] \mathrm{J} \mathrm{m}^{-2} \mathrm{~s}^{-1}
\end{aligned}
$$

where $\alpha_{0}$ is the atmospheric loss cone and $j_{\downarrow}$ and $j_{\uparrow}$ are expressed in units of ions $\mathrm{cm}^{-2} \mathrm{~s}^{-1} \mathrm{sr}^{-1} \mathrm{keV}^{-1}$. For an upward $\mathrm{H}^{+}$ion flux of the order of $10^{7}$, one obtains an upward directed energy flux of the order of $\approx 6.7 \times 10^{-8} \mathrm{~J}$ $\mathrm{m}^{-2} \mathrm{~s}^{-1}$. After taking into account the fact that the flux of upgoing protons is often cut above $40 \mathrm{eV}$ (as is observed when an intermediate energy channel is used), the upgoing kinetic energy flux is lowered to $\approx 5 \times 10^{-8} \mathrm{~J} \mathrm{~m}^{-2} \mathrm{~s}^{-1}$.

As it is likely that we overestimate the mechanical energy fluxes, since the conical shape of the distribution function of the suprathermal protons is not taken into account, we consider that the above described theory gives a satisfying estimate for the energetic transfer that is required to explain the formation of hydrogen conics inferred from AUREOL 3 measurements. 


\section{Summary and Conclusions}

Recent measurements from DE 1 [Crew et al., 1990], Viking [André et al., 1988], and AUREOL 3 [Rauch et al., this issue] have given evidence of a strong correlation between the transverse heating of minor ions and the presence of large-amplitude Alfvénic fluctuations. The present work is an attempt to explain how such downgoing electromagnetic Alfven waves can heat these thermal ions up to perhaps a few hundred electron volts. Motivated by the observational results presented by Rauch et al. [this issue], our analysis has been focused on the case where there is a majority of $\mathrm{O}^{+}$ions. The opposite case, where $\mathrm{H}^{+}$is the main species, will be presented in a forthcoming paper. It has been shown that magnetosonic waves, as they propagate earthward, can be mode converted, via the inhomogeneity of the geomagnetic field, into low phase velocity ion cyclotron waves propagating upward. Since this conversion occurs mainly at the altitude where the wave frequency matches the gyrofrequency of the protons, these waves can resonantly heat these minor $\mathrm{H}^{+}$ions. The resulting resonant absorption process has been studied in detail, in the framework of the cold plasma approximation, which allows an estimate of the reflection, transmission, and absorption coefficients, as a function of the incidence angle and of the relative density of the minor ion. This heating process is likely to occur over a relatively broad altitude range. The cumulative heating, combined with the adiabatic upward motion of the ions, is expected to produce the conical distribution functions at higher altitudes, as suggested by Chang et al. [1986], Retterer et al. [1987], and Crew et al. [1990].

At the low altitudes covered by AUREOL $3, \mathrm{H}^{+}$conics were found in the light ion trough, where these ions provide the minor species of the plasma. This is expected from our theory, which foresees that only minor $\mathrm{H}^{+}$can be efficiently heated by the resonant absorption process. The most prominent feature found in wave data during these heating events is the existence of a narrow peak affecting the spectra of the parallel electric component $E_{z}$, at frequencies lying just below (a few hertz) the proton gyrofrequency. While not important for the transverse heating of $\mathrm{H}^{+}$, this spectral line testifies to the existence of the resonant absorption process. According to our calculations, this component is indeed expected to be strong close to $f_{\mathrm{H}^{+}}$, in the vicinity of the conversion layer. The corresponding spectral width can be roughly estimated by taking into account thermal effects. The need for a low $\mathrm{H}^{+}$concentration is consistent with this signature of the mode conversion being found primarily in the light ion trough region, as firmly established by the data. Finally, it has been shown that the electromagnetic energy flux that can be extracted from the incident waves matches the mechanical energy flux supplied by the upgoing $\mathrm{H}^{+}$ions.

When the $\mathrm{O}^{+}$ions become the minor species, it is expected that they could also be heated by Alfvén waves propagating downward and having frequencies in the $f_{\mathrm{O}^{+}}$ range. Observationally, the oxygen heating process does not seem to have been observed on board AUREOL 3, partly because of the altitude range covered by this satellite and partly because the wave measurements were not sensitive enough close to $f_{\mathrm{O}^{+}}$. Detailed comparisons between measurements effected over a broader altitude range and including waveform measurements of the electric and magnetic components of the waves are required to determine the role played by the process discussed here. Such measurements will be done, in the near future, by the missions Interbal, Freja, and Polar.

\section{APPENDIX}

From equations $\left(2 a^{\prime}\right)$ and $\left(2 b^{\prime}\right)$, one gets, after having performed a Fourier transform in $\xi$,

$$
\begin{gathered}
\left(n^{2}+\alpha\right) E_{x}+i \beta E_{y}=i \eta_{\mathrm{H}^{+}} \int\left(E_{x}+i E_{y}\right) d n \\
-i \beta E_{x}+\left(n^{2}+n_{\perp}^{2}+\alpha\right) E_{y}=\eta_{\mathrm{H}^{+}} \int\left(E_{x}+i E_{y}\right) d n
\end{gathered}
$$

where $\alpha=1 / 255, \beta=16 / 255$, and $\beta-\alpha=1 / 17$. After having performed linear combinations of these two equations, one gets

$$
\begin{gathered}
E_{x}=i \frac{n^{2}+n_{\perp}^{2}-(1 / 17)}{n^{2}-(1 / 17)} E_{y} \\
P_{\infty}(n) E^{+}=2 i \eta_{\mathrm{H}^{+}} Q(n) \int E^{+}(n) d n
\end{gathered}
$$

where $P_{\infty}(n)$ and $Q(n)$ are polynomials, given by

$$
\begin{gathered}
P_{\infty}(n)=\left(n-n_{+}\right)\left(n-n_{-}\right)\left(n+n_{-}\right)\left(n+n_{+}\right) \\
Q(n)=n^{2}+n_{\perp}^{2} / 2-1 / 17
\end{gathered}
$$

with $n_{ \pm}^{2}= \pm \Delta^{1 / 2}-\left(1 / 255+n_{\perp}^{2} / 2\right)$ and $\Delta=(16 / 255)^{2}+$ $n_{\perp}^{4} / 4 . E^{+}(n)$ is the Fourier transform of the left-handed component of the electric field $\left(E^{+}=E_{x}+i E_{y}\right)$. As expected, the negative mode is nonpropagating for $\|\xi\| \rightarrow \infty$ (Figure A1).

From (A2) the function $H(n)=P_{\infty}(n) Q^{-1}(n) E^{+}(n)$ can be determined:

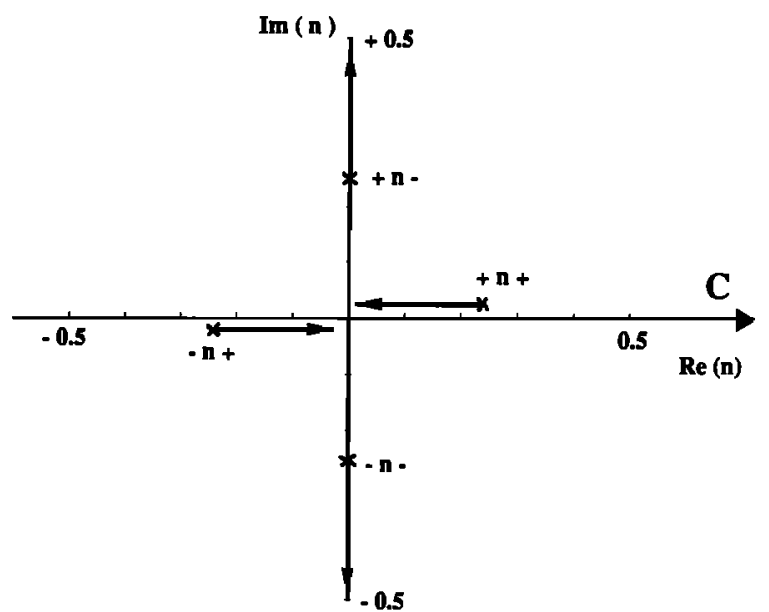

Fig. Al. Position of the poles of the integrand defining $\Psi$. The arrows show their motion in the complex plane, for increasing $n_{\perp}$. The $\pm n_{+}$must have a small imaginary part to be consistent with causality requirements. For $\xi<0$ the integration path $\mathscr{C}$ can be chosen along the real axis. Closing this path in the negative imaginary plane, where the integral defining $\Psi$ is convergent, ensures that only downgoing waves contribute, as required from the chosen boundary conditions. 


$$
H(n)=\exp \left\{2 i \eta_{\mathrm{H}^{+}} \int \frac{Q(n)}{P_{\infty}(n)} d n\right\}
$$

where $\eta_{\mathrm{H}^{+}}$is defined in section 3.1. Carrying out the integral in the right-hand side of (A3), one gets finally

$$
E^{+}(n)=\left(n^{2}+\frac{n_{\perp}^{2}}{2}-\frac{1}{17}\right)[G(n)]^{-1}
$$

with

$$
\begin{aligned}
& G(n)=\left(n-n_{+}\right)^{1-i \delta_{+}}\left(n-n_{-}\right)^{1-i \delta_{-}} \cdot\left(n+n_{-}\right)^{1+i \delta_{-}\left(n+n_{+}\right)^{1+i \delta_{+}}} \\
& \delta_{ \pm}=\eta_{\mathrm{H}^{+}} \frac{Q\left(n_{ \pm}\right)}{2(\Delta)^{1 / 2} n_{ \pm}}=\eta_{\mathrm{H}^{+}}\left( \pm \Delta^{1 / 2}-\frac{16}{255}\right) \\
& \cdot\left\{2(\Delta)^{1 / 2}\left[ \pm \Delta^{1 / 2}-\left(\frac{1}{255}+\frac{n_{\perp}^{2}}{2}\right)\right]^{1 / 2}\right\}^{-1}
\end{aligned}
$$

Notice that $\delta_{+}$is real whenever $\delta_{-}$is purely imaginary. Now, taking the inverse Fourier transform of (A4), the function $E^{+}(\xi)$ can be obtained by applying the differential operator $\left(n_{\perp}^{2} / 2-1 / 17-d^{2} / d \xi^{2}\right)$ to the function $\Psi(\xi, \mathscr{C})$, defined in equation (7). Similarly, from equations $(1 a)-(1 d)$ the other components of the electromagnetic field can easily be determined (see equations $(8 a)-(8 c)$ ). The poles $n_{ \pm}$of the integrand appearing in the definition of $\Psi(\xi, \mathscr{C})$ are sketched in Figure A1. Notice that we have given a small negative (positive) imaginary part to the pole that has a negative (positive) real part. When finite- $\varepsilon_{\mathbf{H}^{+}}$corrections are retained in equations $\left(2 a^{\prime}\right)$ and $\left(2 b^{\prime}\right)$, similar calculations can be made which lead to slightly more complex expressions for $n_{ \pm}$and $\delta_{ \pm}$:

$$
\begin{aligned}
n_{+}^{2}=\Delta^{1 / 2}-\left\{\frac{n_{\perp}^{2}}{2}+\frac{1}{255}\left(1-\frac{319}{64} \varepsilon_{\mathrm{H}^{+}}\right)\right\} \quad(\mathrm{A} 5 \\
\delta_{+}=\eta_{\mathrm{H}^{+}}\left[\Delta^{1 / 2}-\frac{16}{255}\left(1-\frac{769}{1024} \varepsilon_{\mathrm{H}^{+}}\right)\right] \\
\cdot\left\{2(\Delta)^{1 / 2}\left[\Delta^{1 / 2}-\left(\frac{n_{\perp}^{2}}{2}+\frac{1}{255}\left(1-\frac{319}{64} \varepsilon_{\mathrm{H}^{+}}\right)\right)\right]\right\}^{-1}
\end{aligned}
$$

where

$$
\Delta=\frac{n_{\perp}^{4}}{4}+\left(\frac{16}{255}\right)^{2}\left(1-\frac{769}{1024} \varepsilon_{\mathrm{H}^{+}}\right)^{2}
$$

The contour $\mathscr{C}$ has to take into account the boundary conditions of the problem. It must respect the causality condition and ensure the convergence of the solutions over the whole $\xi$ axis. Let us first consider the wave propagation "at infinity," far from the coupling points. After having performed a Laplace (in time) and a Fourier (in space) transform, the Green's function of the propagation problem has to be determined by integrating the transform over a Brownwich path lying high in the upper half plane of the complex variable $\omega$, and on a Fourier contour lying, in the complex $k$ plane, between the various singularities of the dispersion equation [Bers, 1972]. These singularities appear for $k= \pm(\omega / c) n_{ \pm}(\infty)$, such that positive values of $\operatorname{Re}(k)$
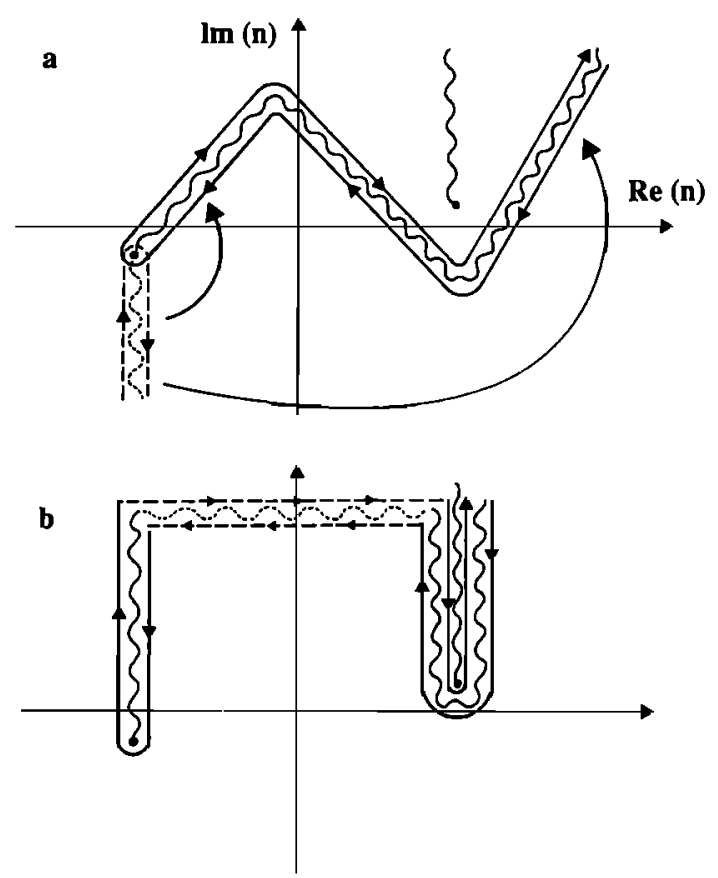

Fig. A2. (a) For $\xi<0$, path $\mathscr{C}$ can be downshifted into the negative imaginary plane; then the contribution of each pole can be determined by an integration over an inverse U-shaped path encircling it (dashed line at lower left). Here one can neglect the contribution of the purely imaginary pole, which gives only a nonpropagative contribution. In order to properly match the solution from $\xi<0$ to $\xi>0$, one has to rotate the path by an angle $+\pi$, without traversing the branch cut (wavy lines) issuing from the poles. This is indicated by the two kinks given to the path (full line). (b) Final configuration of $\mathscr{C}$, for $\xi>0$. The U-shaped path on the left corresponds to the incident waves, while the two paths on the right correspond to the reflected waves (see text).

correspond to waves propagating in the $+z$ direction while negative values of $\operatorname{Re}(k)$ correspond to waves which propagate in the $-z$ direction. To take into account causal requirements, this implies that any pole with positive (negative) real value must be considered as having a positive (negative) imaginary part, as drawn in Figure A1. Therefore in expression (7) the integration path $\nvdash$ must first be chosen on the real $n$ axis above the poles $-n_{ \pm}$and below the poles $+n_{ \pm}$(Figure Al).

In the present case, the factor containing the imaginary poles $\pm n_{-}$, in the denominator of the integrand in (7), will contribute negligibly to the value of $\Psi(\xi, \mathscr{C})$, as compared to the divergent terms containing the propagating poles $\pm n_{+}$. Thus the integral can be simplified by keeping only the poles corresponding to propagation waves:

$$
\Psi(\xi, \mathscr{C}) \approx \int_{\mathscr{C}} \frac{\exp (\operatorname{in} \xi) d n}{\left(n+n_{+}\right)^{1+i \delta_{+}}\left(n-n_{+}\right)^{1-i \delta_{+}}}
$$

Now, for $\xi<0, \mathscr{C}$ can be translated down into the half plane $\operatorname{Im}(n)<0$ (the integrand being then convergent), in such a way that the poles $-n_{+}$, and the corresponding branch cut, are encircled by the new path (dashed curve at lower left in Figure A2a). Only downgoing waves with refraction indices $n_{+}$are thus expected to be found asymptotically, for $\xi \rightarrow-\infty$, which corresponds to the chosen boundary conditions. Thus, for $\xi<0$, the integration path must be chosen such that on $\mathscr{G}, n=-n_{+}+\rho e^{i \theta}$, with $\theta=$ 
$-\pi / 2$ or $-5 \pi / 2$, and $\rho$ varies from 0 to $+\infty$. To match these expressions, valid for $\xi<0$, with those valid for $\xi>0$ and offer a single solution of (A2), one has to change in a continuous way the path $\mathscr{C}$, such that the integral appearing in (7) (or (A5)) always converges and that $\mathscr{C}$ never cuts any branch cut nor crosses any poles. According to the "Budden matching rule" [Budden, 1966, p. 477], in order to "go from $\xi=-\infty$ to $\xi=+\infty$," one has to rotate the variable $\xi$ in the complex plane such as to keep Im $(\xi)>0$; thus one must go from $\arg (\xi)=+\pi$ to $\arg (\xi)=0$. For $\Psi(\xi, \mathscr{b})$ to be always defined by a convergent integral, this implies a corresponding rotation of $+\pi$ of the integration paths, as shown in Figures $\mathrm{A} 2 a$ and $\mathrm{A} 2 b$, where we have decomposed the transformation into two stages to show how the poles and branch cuts have to be avoided. The integrals over the path lying on the negative real half plane correspond to downgoing or incident waves, while those over the paths lying on the positive half plane correspond to the upgoing, reflected waves. One has to take into account that on the internal $\mathrm{U}$-shaped path the argument of the function $\left(n+n_{+}\right)$has been rotated by $-2 \pi$ with respect to its value on the external path. From equation (A6) these various integrals can be explicitly defined as confluent hypergeometric functions [Abramowitz and Stegun, 1970, chapter 13.2, equation 13.2.8]. Finally, up to an arbitrary multiplicative constant the solution is written

$$
\begin{gathered}
\Psi_{T}=e^{i \phi} U\left(-i \delta_{+}, 0,2 n_{+} i \xi\right) e^{-i n_{+} \xi} \\
\arg (\xi)=+\pi \\
\Psi_{I}=U\left(-i \delta_{+}, 0,2 n_{+} i \xi\right) e^{-i n_{+} \xi} \quad \arg (\xi)=0 \\
\Psi_{R}=e^{i \psi}\left\{1-e^{-2 \pi \delta_{+}}\right\} U\left(i \delta_{+}, 0,-2 n_{+} i \xi\right) e^{i n_{+} \xi}
\end{gathered}
$$

for the transmitted, incident, and reflected waves, respectively, where $U(a, b, z)$ is the irregular Kummer's function [Abramowitz and Stegun, 1970, chapter 13]. The $\phi$ and $\psi$ phase shifts allow the continuity of the solution $\left(\Psi_{T}=\Psi_{l}+\right.$ $\Psi_{R}$ at $\xi=0$ ):

$$
\begin{gathered}
\sin \left(\frac{\phi}{2}\right)=\frac{1}{2}\left(1-e^{-2 \pi \delta_{+}}\right) \\
\Psi=\frac{1}{2}\left[\pi+\phi-2 \arg \left(\frac{\Gamma\left(1-i \delta_{+}\right)}{\Gamma\left(1+i \delta_{+}\right)}\right)\right]
\end{gathered}
$$

The "amplification" factor is written

$$
\frac{\|\Psi(0)\|^{2}}{\|\Psi(+\infty)\|^{2}}=\frac{e^{\pi \delta_{+}} \sinh \left(\pi \delta_{+}\right)}{\pi \delta_{+}\left\|1+e^{i \psi}\left(1-e^{-2 \pi d_{+}}\right)\right\|^{2}}
$$

From the asymptotic expansion of the Kummer's functions, and taking into account that the Poynting flux vector has the same expression as a function of $\Psi$, for $\xi \rightarrow \pm \infty$ :

$$
S_{\xi} \propto n\left\{\left(n^{2}+n_{\perp}^{2}-1 / 17\right)^{2}+\left(n^{2}-1 / 17\right)^{2}\right\}\|\Psi\| 2
$$

one gets the expressions (11), for the transmission, reflection, and absorption coefficients. Finally,

$$
U\left(i \delta_{+}, 0,2 i n_{+} \xi\right) \approx 1 / \Gamma\left(1+i \delta_{+}\right)+O\left(\left|2 n_{+} \xi \ln \left(2 n_{+} \xi\right)\right|\right)
$$

for $\xi \rightarrow 0$, where $\Gamma$ means the usual $\Gamma$ function [Abramowitz and Stegun, 1970, equation 13.5.11]. $\Psi(\xi, \mathscr{C})$ is thus finite at $\xi \rightarrow 0$, but its first, second, and third derivatives here diverge like $\ln (\xi), \xi^{-1}$, and $\xi^{-2}$, respectively.

Acknowledgments. The Editor thanks the two referees for their assistance in evaluating this paper.

\section{REFERENCES}

Abramowitz, M., and I. A. Stegun, Handbook of Mathematical Functions, Dover, New York, 1970.

André, M., H. Koskinen, L. Matson, and R. Erlandson, Local transverse ion energization in and near the polar cusp, Geophys. Res. Lett., 15, 107, 1988.

Ashour-Abdalla, M., and H. Okuda, Turbulent heating of heavy ions on auroral field lines, J. Geophys. Res., 89, 2235, 1984.

Ball, L., Can ion acceleration by double-cyclotron absorption produce $\mathrm{O}^{+}$ion conics?, J. Geophys. Res., 94, 15257, 1989.

Bers, A., Linear waves and instabilities, in Plasma Physics, edited by C. De Witt and J. Peyraud, p. 113, Gordon and Breach, New York, 1972.

Budden, K. G., Radio Waves in the Ionosphere, Cambridge University Press, New York, 1966.

Burch, J. L., Energetic particles and currents: Results from Dynamics Explorer, Rev. Geophys., 26, 215, 1988.

Cairns, R. A., and C. N. Lashmore-Davies, A unified theory of a class of mode conversion problems, Phys. Fluids, 26, 1268, 1983.

Chang, T., and B. Coppi, Lower hybrid acceleration and ion evolution in the suprauroral region, Geophys. Res. Lett., 8, 1253, 1981.

Chang, T., G. B. Crew, N. Hershkowitz, J. R. Jasperse, J. M. Retterer, and J. D. Winningham, Transverse acceleration of oxygen ions by electromagnetic ion cyclotron resonance with broad band left-hand polarized waves, Geophys. Res. Lett., 13, $636,1986$.

Crew, G. B., and T. Chang, Asymptotic theory of ion conic distributions, Phys. Fluids, 28, 2382, 1985.

Crew, G. B., and T. Chang, Path integral formulation of ion heating, Phys. Fluids, 31, 3425, 1988.

Crew, G. B., T. Chang, J. M. Retterer, W. K. Peterson, D. A. Gurnett, and R. L. Huff, Ion cyclotron resonance heated conics: Theory and observations, J. Geophys. Res., 95, 3959, 1990.

Dusenbery, P. B., and L. R. Lyons, Generation of ion conic distributions by upgoing ionospheric electrons, J. Geophys. Res., $86,7627,1981$.

Fuchs, V., K. Ko, and A. Bers, Theory of mode-conversion in weakly inhomogeneous plasma, Phys. Fluids, 24, 1251, 1981.

Ginzburg, V. L., The Propagation of Electromagnetic Waves in Plasmas, Pergamon, New York, 1970.

Gurnett, D. A., R. L. Huff, J. D. Menietti, J. L. Burch, J. D. Winningham, and S. D. Shawhan, Correlated low-frequency electric and magnetic noise along auroral field lines, J. Geophys. Res., 89, 8971, 1984.

Johnson, J. R., T. Chang, and G. B. Crew, Mode conversion of low frequency waves in a multispecies plasma (abstract), Eos Trans. $A G U, 69,1375,1988$.

Johnson, J. R., T. Chang, G. B. Crew, and M. André, Equatorially generated ULF waves as a source for the turbulence associated with ion conics, Geophys. Res. Lett., 16, 1469, 1989.

Kintner, P. M., M. C. Kelley, and F. S. Mozer, Electrostatic hydrogen cyclotron waves near one earth radius altitude in the polar magnetosphere, Geophys. Res. Lett., 5, 139, 1978.

Klumpar, D. M., A digest and comprehensive bibliography on transverse auroral ion acceleration, in Ion Acceleration in the Magnetosphere and Ionosphere, Geophys. Monogr. Ser., vol. 38, edited by T. Chang, p. 389, AGU, Washington, D. C., 1986.

Lashmore-Davies, C. N., V. Fuchs, and R. A. Cairns, Ion-cyclotron resonance heating by means of the fast wave in a longitudinally inhomogeneous magnetic field, Phys. Fluids, 28, 1791, 1985.

Mozer, F. S., C. A. Cattell, M. Temerin, R. B. Torbert, S. von Glinski, M. Woldorff, and J. Wygant, The dc and ac electric field, plasma density, plasma temperature, and field-aligned current 
experiments on the S3-3 satellite, J. Geophys. Res., 84, 5875, 1979.

Rauch, J. L., and A. Roux, Ray tracing of ULF waves in a multicomponent magnetospheric plasma: Consequences for the generation mechanism of ion cyclotron waves, J. Geophys. Res., 87, 8191, 1982.

Rauch, J. L., F. Lefeuvre, D. Le Quéau, A. Roux, J. M. Bosqued, and J. J. Berthelier, Heating of protons by resonant absorption in a multicomponent plasma, 1, Experimental evidence, J. Geophys. Res., this issue.

Retterer, J. M., T. Chang, and J. R. Jasperse, Ion acceleration by lower hybrid waves in the suprauroral region, J. Geophys. Res., 91, 1609, 1986.

Retterer, J. M., T. Chang, G. B. Crew, J. R. Jasperse, and J. D. Winningham, Monte Carlo modeling of ionospheric oxygen acceleration by cyclotron resonance with broad band electromagnetic turbulence, Phys. Rev. Lett., 59, 148, 1987.

Sharp, R. D., R. G. Johnson, and E. G. Shelley, Observation of an ionospheric acceleration mechanism producing energetic (keV) ions primarily normal to the geomagnetic field direction, $J$. Geophys. Res., 82, 3324, 1977.

Smith, R. L., and N. M. Brice, Propagation in multicomponent plasmas, J. Geophys. Res., 69, 5029, 1964.

Stix, T. H., The Theory of Plasma Waves, McGraw-Hill, New York, 1962.

Taylor, H. A., Jr., and W. J. Walsh, The light-ion trough, the main trough, and the plasmapause, J. Geophys. Res., 77, 6716, 1972.
Temerin, M., and I. Roth, Ion heating by waves with frequencies below the ion gyrofrequency, Geophys. Res. Lett., 13, 1109, 1986.

Ungstrup, E., D. M. Klumpar, and W. J. Heikkila, Heating of ions to superthermal energies in the topside ionosphere by electrostatic cyclotron waves, J. Geophys. Res., 84, 4289, 1979.

White, R. B., S. Yoshikawa, and C. Oberman, Alfven wave cyclotron resonance heating, Phys. Fluids, 25, 384, 1982.

Winningham, J. D., and J. L. Burch, Observation of large-scale ion conic generation with DE-1, in Physics of Space Plasmas (19824), SPI Conf. Proc. Reprint Ser., vol. S, edited by J. Belchẹr, $\mathbf{H}$. Bridge, T. Chang, B. Coppi, and J. R. Jasperse, Scientific, Cambridge, Mass., 1984.

J. M. Bosqued, CESR/CNRS, 9 Avenue du Colonel Roche, F-31029 Toulouse Cedex, France.

F. Lefeuvre and J. L. Rauch, LPCE/CNRS, 3A Avenue de la Recherche Scientifique, F-45071 Orléans Cedex 02, France.

D. Le Quéau, Entité CNRS du CRPE, 10, 12 Avenue de l'Europe, 78140 Vélizy, France.

A. Roux, CRPE/CNET-CNRS, 92131. Issy-les-Moulineaux Cedex France.

(Received October 29, 1990; revised July 3, 1991; accepted August 16, 1991.) 